\title{
A novel mutation in the ATP1A2 gene causes alternating hemiplegia of childhood
}

\author{
M T Bassi, N Bresolin, A Tonelli, K Nazos, F Crippa, C Baschirotto, C Zucca, A Bersano, D Dolcetta, \\ F M Boneschi, V Barone, G Casari
}

A Iternating hemiplegia of childhood (AHC, MIM 104290) is a rare syndrome, characterised by early onset of episodic hemi- or quadriplegia lasting minutes to days. This disorder, first reported by Verret and Steel in $1971,{ }^{1}$ has historically been thought to represent a migraine equivalent ${ }^{1}$ or an unusual form of epilepsy or a movement disorder, ${ }^{2}$ as it typically presents with complex and variable clinical features.

In most patients, the earliest manifestations clearly related to AHC are tonic-dystonic attacks and paroxysmal nystagmus associated with autonomic changes and paroxysmal dyspnoea and usually appear between 3 and 6 months of age. The hemiplegic episodes develop before 18 months of age lasting anywhere from minutes to days at a time and involving either side of the body or shifting from one side to the other during the same episode with a period of bilateral weakness when the second side becomes involved. ${ }^{3}$

Some attacks are characterised by a bilateral involvement, which is apparent from the beginning, and do not follow an hemiplegic episode, ${ }^{3}$ which determines extreme hypotonia of the whole body with inability to move and a level of consciousness markedly depressed.

A characteristic feature of AHC is the disappearance of all abnormalities when the child falls asleep. ${ }^{3}$

With increasing age, hemiplegic episodes follow a general pattern of initially increasing frequency and duration, followed by a plateau, and finally by a decrease in the number and duration of attacks. Analogously, the paroxysmal manifestations associated with hemiplegia tend to decrease in frequency and intensity with time and usually disappear after 5-7 years. Development before the onset of hemiplegic episodes may be normal or delayed.

Epileptic seizures are reported in a variable percentage of sporadic patients. ${ }^{3-5}$

An association with migraine has also been noticed since the earliest description. ${ }^{1}$ Indeed, for some time, the international classification of headaches (IHC) has included AHC as a childhood periodic syndrome that may be associated with complicated migraine (familial hemiplegic migraine, FHM), ${ }^{6}$ mostly due to the clinical similarity of the attacks and the paroxysmal nature of both disorders. Some of the aspects of AHC, however, are clearly distinct from FHM, including choreoathetosis, dystonic posturing, and a progressive course associated with mental deterioration. ${ }^{7}$ Concerning the possible genetic origin of the disease, only a few familial cases have been described so far, providing evidence to support an autosomal dominant genetic mechanism of transmission. ${ }^{8}{ }^{9}$ In the first AHC family described, a balanced reciprocal translocation was detected: 46,XY, t(3;9)(p26;q34), thus indicating the first two genomic regions possibly associated

\section{Key points}

- Alternating hemiplegia of childhood (AHC, MIM 104290) is a rare syndrome, characterised by early onset of episodic hemi- or quadriplegia lasting minutes to days. The majority of patients are sporadic. Only a few familial cases are reported in the literature.

- Here we describe a new familial case from a Greek island with four affected members in two generations, the mother and three out of four children. All patients share a normal karyotype. Due to the partial clinical overlap of familial hemiplegic migraine (FHM) with $A H C$, we screened the ATPIA2 gene coding for the $\alpha 2$ subunit of the $\mathrm{Na}, \mathrm{K}$ pump, associated with $\mathrm{FHM}$ type 2.

- We found a novel heterozygous mutation segregating with the disease and causing a threonine to asparagine replacement (T378N). This missense mutation localises to the ATPases phosphorylation site of the hydrolase domain. The affected residue is highly conserved in all the known $\alpha$ subunits of the $\mathrm{Na}, \mathrm{K}$ and $\mathrm{Na}, \mathrm{H}$ pumps from vertebrates to invertebrates. Functional data suggest that loss of function of the mutated ATPIA2 isoform is involved in generating the disease phenotype. This is the first mutation associated with $\mathrm{AHC}$ identified so far.

with the disease. ${ }^{8}$ Subsequently, mitochondrial abnormalities identified in a few sporadic AHC patients suggested the hypothesis, which was however never clearly confirmed, of a mitochondrial dysfunction underlying the pathogenesis of the disease. ${ }^{10}$

In the present study, we fully describe a new AHC family from a Greek island, with four affected members in two generations. Molecular genetic analysis of the family allowed us to identify a single novel mutation in the ATPIA2 gene, segregating in all affected individuals. The gene maps on chromosome 1q23 and encodes for the $\alpha 2$ subunit of the

Abbreviations: $A H C$, alternating hemiplegia of childhood; $B F I C$, benign infantile convulsions; CK, creatin kinase; CT, computed tomography; EEG, electroencephalography; FHM, familial hemiplegic migraine; IHC, international classification of headaches; MRI, magnetic resonance imaging 
plasma membrane $\mathrm{Na}, \mathrm{K}$ pump, a heteromeric protein essential for all mammalian cells that consumes ATP to actively transport $\mathrm{Na}^{+}$out and $\mathrm{K}^{+}$into the cell. Mutations in ATPIA2 are involved in generating familial hemiplegic migraine type II disease (FHM2) either isolated ${ }^{11}$ or in association with benign infantile convulsions (BFIC). ${ }^{12}$ The new mutation identified in our AHC family causes an amino acid change $(\mathrm{T} 378 \mathrm{~N})$ falling in the protein hydrolase domain within the consensus site for phosphorylation of the $\mathrm{Na}, \mathrm{K}$ ATPases. Functional data indicate that this mutation has a loss of function effect although the protein results in the correct plasma membrane location.

This mutation represents the first molecular change associated with the AHC syndrome.

\section{METHODS}

\section{Case presentation}

The pedigree of the AHC family represented in fig 1 shows four affected members in two generations, the mother (II-4) and three out of four children (III-1, III-3, III-4). The second son (individual III-2) is a healthy boy with normal neuropsychological and neuromotor development. The family has been ascertained by two of the authors (NB and KN). The clinical features of the affected members are quite different suggesting high phenotype variability within this family. A blood sample from individual I-3 (deceased) was not available. However, all members of the family describe him as a healthy man throughout his life with no signs or symptoms related to the disease.

\section{Individual II-4}

The subject is a 38 year old Caucasian woman, delivered at term after a trouble-free pregnancy. She presented some delay in acquiring language skills, but apparently experienced no other problems in achieving developmental milestones. At the age of 2 years, she presented the first attack of alternating hemiplegia; the episode lasted $24 \mathrm{~h}$ and was apparently evoked by a head trauma secondary to a fall from the stairs. During the attack, the hemiplegia shifted sides, and during the transition she became tetraparetic and unable to eat or talk. Sleep was associated with resolution of the attack. The resolution persisted for 15-20 min after awakening and was followed by recurrence of the symptoms during wakefulness. Because of this attack, she was hospitalised for 20 days, but a complete neuroradiological examination was never performed. After this first major episode, she suffered only mild hemiplegic attacks, occurring sporadically mostly in adulthood, during pregnancy, and also later, the last having occurred at 38 years of age. No autonomic disturbances were referred during or between hemiplegic attacks. In addition, in the years following the first episode, progressive cognitive deterioration was referred. She had no clinical history of seizures or headache and there were no familial cases of migraine. At the last neurological examination (performed at 42 years of age), choreoathetoid movements and horizontal nystagmus were present with mild gait ataxia. Mild spasticity was noticed and a slight left-side prevalence of tendon reflexes $(2+)$; plantar responses were bilaterally flexor. The patient shows mild dysmetria and intention tremor at cerebellar functional tests. Cranial nerve examination was normal as well as position and sensations. At the time of hospitalisation and AHC diagnosis of the first affected son (individual III-1), muscle biopsy was performed and was normal.

At 38 years of age, she was mildly mentally retarded with a persistent disability characterised by difficulty in understanding and performing even simple orders, a limited fund of knowledge, literal interpretation, and lack of understanding of proverbs. However, a complete formal neuropsychological evaluation was never carried out.

\section{Subject III- 1}

The subject is a 12 year boy, born at full term after an uneventful pregnancy and normal delivery. There was no
।

II

I
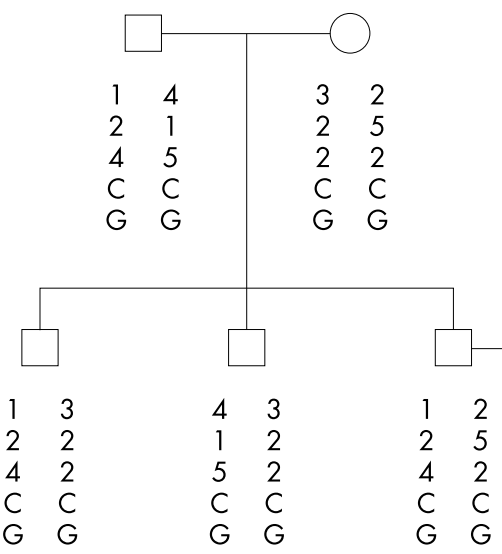

$\begin{array}{ll}4 & 3 \\ 1 & 2 \\ 5 & 2 \\ C & C \\ G & G\end{array}$

$\begin{array}{ll}1 & 2 \\ 2 & 5 \\ 4 & 2 \\ C & C \\ G & G\end{array}$

\begin{tabular}{c|c}
2 & 3 \\
3 & 4 \\
$\operatorname{la}$ & 2 \\
$A^{\star}$ & $C$ \\
$A$ & $G$
\end{tabular}
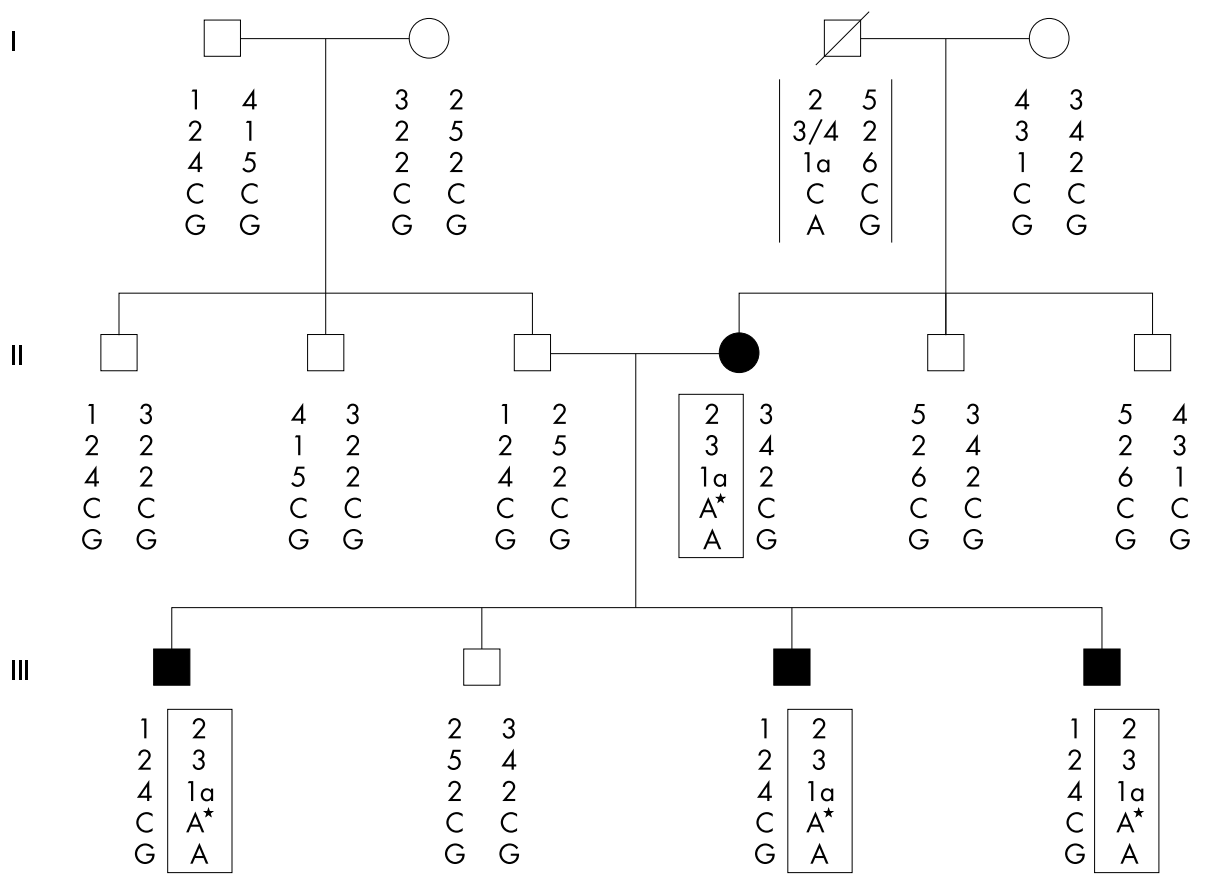

Figure 1 Pedigree of the AHC family. The figure shows the FHM2 locus haplotype (markers are D1S2707, NIB1 152, FHM2-CA, ATP1A2/1237-C/ $A^{*}$, and ATP1A2/IVS19-G/A, from top to bottom, respectively). The star indicates the base change in all affected members. Disease haplotype is boxed, while markers within open bars (individual I-3) refer to the inferred haplotype of the maternal grandfather. 
neonatal distress. Delayed physical development was referred: he was able to sit at 8 months of age, to stand at 12 months, to walk independently at 16 months and to have sphincter control at the age of 3 years. At 3 years, a neuropsychological evaluation revealed significant developmental delay with an overall functional level according to the Stanford Binet Intelligence Scale of a 2 year old child. He was referred as an "aggressive child" with semantic paraphasia.

At 2 years of age, an episode of loss of consciousness lasting 20 min was reported. Immediately after this episode, repeated bouts of hemiplegia of varying intensity involving either side of the body were reported. During most of the attacks, the patient presented with flaccid hemiplegia shifting sides, sometimes associated with tremor mostly involving upper limbs. Each of these attacks was associated with paroxysmal phenomena including dystonic posturing, which often appeared intermittently during the hemiplegic events. Choreoathetoid movements, nystagmus, and eye deviation were also seen during and between hemiplegic attacks, but no autonomic disturbances or history of seizure were reported. He never complained about headache during the attacks. All attacks were relieved by sleep.

Because of the first episode at 2 years of age, he was admitted to hospital and a computed tomography (CT) scan was performed, which was normal, and an electroencephalography (EEG) recording carried out during wakefulness and sleep. The EEG recording showed only mild asymmetry of the background activity with a focal slowing over central and centrotemporal regions predominating on the left side. The frequency of the subsequent attacks was approximately once to twice monthly, mostly evoked by emotional stress or by mild head trauma, the last happening a few months before the last neurological exam which was performed at the age of 12 years. At that time, mild increased tone was found, tendon reflexes were $3+$ throughout, and a right extensor plantar response and a bilateral achilleus clonus were present.

$\mathrm{He}$ is now clearly mentally retarded with lack of understandings of simple words or simple commands and an overall phenotype indicating severe neurological impairment.

\section{Subject III-3}

The subject is the third son, a 10 year old boy, born after a normal pregnancy and delivery. He presented delayed physical and mental development: he sat at 15 months, took his first steps almost at the same age, and achieved sphincter control between 2 and 3 years of age. His history included an episode of flaccid hemiparesis involving the left side with eye deviation at the age of 2-3 months. Afterwards, serial episodes of generalised hypotonia were referred, and in the next few years several episodes of flaccid areflexic paralysis of the upper and lower right and left limbs occurred of variable duration, one of 30 days, one of 15 days, and the others of shorter time. The hemiplegic attacks were never associated with headaches. Some attacks were accompanied by dystonic posturing, choreoathetoid movements, nystagmus, and autonomic disturbances such as alteration in skin colour (pale), increased body temperature, and heavy sweating which more rarely also appeared between the attacks. Episodes were dramatically relieved by sleep, or in some cases by submerging the hemiplegic boy in cold water.

Moreover, about three sporadic episodes of generalised tonic-clonic seizures were referred during the patient's life.

Due to all these neurological problems, when he was 2 years old, the child was admitted to hospital where brain magnetic resonance imaging (MRI), CT scan, and magnetic resonance angiography were performed and resulted negative. EEG presented generalised slow diffuse waves. Lactic acid levels were normal, while creatin kinase levels (CK) were increased (624 IU); therefore, a muscle biopsy was performed and reported as normal. A psychologist defined him as a very aggressive child, and a treatment with flunarizine conducted for 2 years apparently worsened his behaviour without any amelioration in the frequency or severity of the attacks. He has not had any hemiplegic bouts since the age of 6 years.

Neurological examination at 10 years of age revealed a general clumsiness, saliva dripping, ataxic gait, and dysarthria as well as nystagmus and mild convergent eye squint. Tendon reflexes were $2+$ at both upper limbs and $3+$ at the lower limbs; plantar responses were flexor bilaterally. Overall, the phenotype shown by the subject is quite similar to that displayed by his brother (individual III-1), as he is clearly mentally retarded, with a limited fund of knowledge, lack of understandings of simple words or simple commands, and characteristic echolalia.

\section{Subject III-4}

The subject is an 8 year old boy, born after a normal pregnancy and delivery. He presented delayed physical and mental development: he was able to sit at 15 months of age and started to walk independently at 18-20 months of age. Sphincter control was obtained at 3 years of age. At the age of 20 months he presented the first episode of flaccid areflexic paralysis involving both sides of the body, which lasted for a few hours, with no preceding seizures. In the following years, he presented a few rare episodes of generalised hypotonia and limpness while fully awake. Usually the attacks started with neck hypotonia followed by trunk muscle weakness and limb hypotonia. The sequence lasted several minutes and, during these episodes, the child had difficulty swallowing and eating and showed facial amimia. During and between these episodes autonomic disturbances were noticed similar to those seen in his oldest brother, but never nystagmus or other ocular abnormalities. No neuroradiological examination was performed. At the last neurological examination (at 7 years of age), there was evidence of mild mental retardation with learning disabilities and mild dysarthria but no other neurological signs.

\section{DNA extraction}

Following informed consent and approval by the ethical committee of the Scientific Institute E. Medea, blood samples were taken from all affected and non-affected members of the family and from 10 Italian sporadic AHC patients belonging to the Italian Association of AHC Syndrome (AISEA). Total DNA was extracted from $5 \mathrm{ml}$ of blood with the IsoQuick Nucleic Acid Extraction kit (ORCA Research, Bothell, WA). A total of 125 healthy individuals ( 50 from the Greek population and 75 from the Italian population) were recruited as control subjects.

\section{Karyotype and haplotype analysis}

Karyotype analysis performed by standard procedure on all affected members of the family gave normal results.

Fluorescence-labelled oligonucleotides for polymorphic markers from the FHM2 locus and from $3 p$ and $9 q$ regions were designed based on the sequences available at the Human Genome Browser. Markers from 3p and 9q genomic regions were chosen to be approximately $1.5 \mathrm{Mb}$ apart. PCR reactions were performed according to the melting temperature of each primer set used. PCR products were detected using an Applied Biosystems ABI 310 PE fluorescent sequencer and analysed with Genescan Analysis 
A

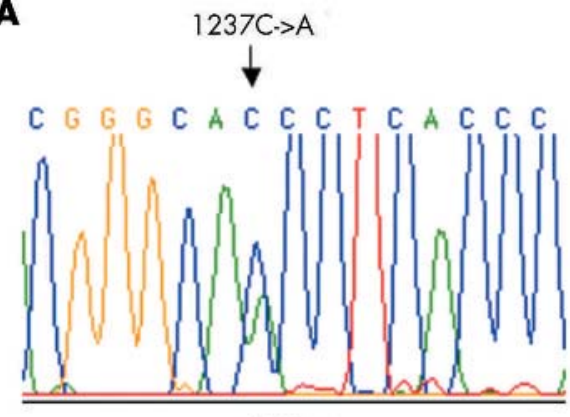

AHC $\mathrm{pt}$

B

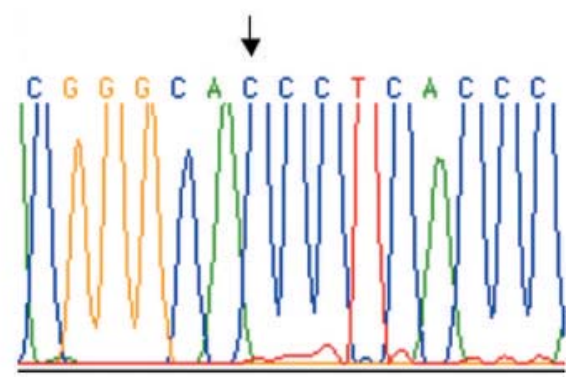

C

Figure 2 Sequence of DNA fragments containing the mutation identified and local amino acid sequence alignment of ATPases. (A) Electropherograms of genomic DNA fragment containing the mutation identified. Arrows show heterozygous 1237C $\rightarrow$ A transversion detected in AHC patients and the corresponding wild type sequence in control DNA. (B) Local amino acid sequence alignment of ATPases. Shown is the complete conservation of the T378 residue (arrow) in several subunits of different Na,K ATPases and H,K ATPases. The accession numbers are indicated on the left, while the amino acid position of the last residue in the alignment is shown on the right for each sequence.

software (version 2.1, Applied Biosystems, Foster City, CA, USA).

\section{Mutation analysis}

Nucleotide numbers refer to the published cDNA sequence (NM 000702). Primers were designed to amplify all 23 exons covering the entire coding region, the exon-intron junctions, and variable portions of introns flanking each exon. Sequences were prepared with a BigDye Terminator Sequencing Kit (version 3.1, Applied Biosystems) run on an Applied Biosystems ABI 3100 Avant Genetic Analyzer and analysed with Sequencing Analysis Software, version 3.7 NT (Applied Biosystems, Foster City, CA, USA).

The mutation carrying fragment was amplified by the following set of primers: forward $5^{\prime}$-GGTCTAGGGTAAGG TTATGG-3'; reverse 5' -CCTCCTCTAATCCTATCCAC-3' .

\section{Constructs and site-directed mutagenesis}

The full-length cDNA coding for the $\alpha 2$ wild-type and ouabain resistant constructs with and without the myc tag were described previously. ${ }^{11}$ We used the QuickChange sitedirected mutagenesis kit (Stratagene, La Jolla, CA, USA) to 
introduce the C1237A mutation in both the myc-tagged and not-tagged ouabain resistant $\alpha 2$ cDNAs.

All constructs were sequence verified.

\section{In vitro transcription and translation}

In vitro transcription and translation was performed using the TNT Coupled Reticulocyte Lysate System (Promega, Madison, WI, USA) and neosynthesised proteins were separated by SDS-PAGE (8\%).

\section{Electrophoresis and Western blot analysis}

Proteins were resuspended in SDS-PAGE buffer $(62.5 \mathrm{mM}$ Tris-HCl pH 6.8, 2\% SDS, 10\% glycerol, 5\% 2-mercaptoethanol) and separated for $2 \mathrm{~h}$ at $100 \mathrm{~V}$ in $8 \%$ SDS-polyacrylamide gels. Transblotted nitrocellulose membranes were incubated with monoclonal primary antibodies anti c-myc 9E10 $(10 \mu \mathrm{g} / \mathrm{ml})$ followed by incubation with horseradish peroxidase (HRP)-conjugated secondary antibodies. Protein bands were visualised with the Enhanced Chemiluminescence Kit (Amersham Biosciences, Piscataway, NJ, USA).

\section{Transfections and ouabain treatment}

Constructs were transfected in HeLa cells by calcium phosphate following standard procedures.

Cell viability was measured with the MTT reduction assay after 24 and $72 \mathrm{~h}$ of $1 \mu \mathrm{M}$ ouabain challenge. We performed two independent experiments and in each experiment datapoints were in triplicate.

\section{Immunocytochemistry}

After transfection $(48 \mathrm{~h})$, COS-7 cells were fixed in $4 \%$ paraformaldehyde and incubated with monoclonal primary antibodies anti c-myc 9E10. Cells were then washed with PBS and incubated with Alexa Fluor 594-conjugated anti-mouse secondary antibody (Molecular Probes, Eugene, OR, USA). Cells were mounted in fluorescent mounting medium (DAKO, Glostrup, Denmark) and visualised under epifluorescence optics.

\section{Subcellular fractionation}

COS-7 cells were lysated on ice in $0.5 \mathrm{M} \mathrm{NaCl}, 10 \mathrm{mM}$ $\mathrm{NA}_{2} \mathrm{CO}_{3}, 0.1 \mathrm{mM}$ PMSF, $10 \mu \mathrm{g} / \mathrm{ml}$ aprotenin, and $10 \mu \mathrm{g} / \mathrm{ml}$ leupeptin, homogenised, and centrifuged at $2000 \mathrm{~g}$ for $20 \mathrm{~min}$ at $4^{\circ} \mathrm{C}$ to discard nuclei and cellular debris. Separation of the membrane fraction (pellet) from the cytosolic fraction (supernatant) was achieved by centrifugation at $100000 \mathrm{~g}$ for $40 \mathrm{~min}$ at $4^{\circ} \mathrm{C}$ in a Beckman TL 100 ultracentrifuge.

\section{RESULTS}

\section{Haplotype analysis and mutation screening}

In order to verify the possible involvement of the genomic regions previously hypothesised by Mikati et al, ${ }^{8}$ namely 3 p26 and $9 \mathrm{q} 34$, in generating the disease, we genotyped all family members with markers spanning a region of $9.5 \mathrm{Mb}$ in 3p25.3-p26 (from D3S1297 to D3S1263) and a region of $24 \mathrm{Mb}$ in 9q32-q34 (from D9S1694 to D9S1838). There was no evidence of common haplotypes being shared by affected individuals.

Due to the clinical overlap of FHM and AHC, we then tested the hypothesis that the gene responsible for FHM type 2, ATP1A2, could be involved in generating the alternating hemiplegia phenotype in this family. The entire family was then genotyped with a small number of microsatellite markers surrounding the FHM2 locus, namely D1S2707, NIB1152, and FHM2 CA, ${ }^{13}$ plus an intragenic single nucleotide polymorphism (SNP) in intron 19. The results shown in fig 1 indicating a possible common haplotype shared by all affected individuals prompted us to screen this family for mutation within this gene. Mutation analysis was performed by direct sequencing of all exons with the same primers used for amplification. A heterozygous mutation $(1237 \mathrm{C} \rightarrow \mathrm{A}$ ) segregating with the disease and causing a threonine to asparagine replacement $(\mathrm{T} 378 \mathrm{~N})$ was found (fig $2 \mathrm{~A}$ ). This mutation is not present in any of the unaffected members of the family and in 250 control chromosomes. Haplotype analysis of all members of the family together with haplotype reconstruction of the maternal grandfather (individual I-3, deceased, see fig 1) supports the hypothesis that the mutation might have arisen in individual II-4 within the chromosome of paternal origin, and then have been transmitted to three out of the four sons.

This missense mutation localises to the ATPases phosphorylation site (DKTGTLT, amino acids 374-380) of the hydrolase domain. In the $\alpha$ subunit topology, the mutated residue resides in the large intracellular loop within the M4-M5 transmembrane segments $(\mathrm{Ml}-\mathrm{Ml})^{14}$. The affected residue is highly conserved in all known $\alpha$ subunits of the $\mathrm{Na}, \mathrm{K}$ and $\mathrm{H}, \mathrm{K}$ pumps from vertebrates to invertebrates (fig 2B) suggesting a functional role in pump activity.

A search for mutations in the same gene was also conducted in a panel of 10 sporadic AHC patients, but the result was negative. As the set of primers used could detect heterozygosity at single nucleotide polymorphic sites present in about half of the introns of the gene (11 out of 23), the results obtained allow us to exclude at least the presence of large deletions encompassing the entire gene in these patients.

\section{Functional analysis of the mutant ATP1A2 protein}

To evaluate the functional consequences of this mutation we carried out transfection experiments in human HeLa cells. Since all mammalian cells have Na,K ATPase, we quenched the endogenous pump activity using ouabain, and tested the function of the exogenously transfected mutant engineered to be resistant to ouabain. Using site-directed mutagenesis we introduced two amino acid changes (Q116R and N127D) in the first extracellular loop to confer resistance to ouabain, and the AHC mutation T378N. HeLa cells transfected with this construct did not survive to $1 \mu \mathrm{M}$ ouabain treatment. The simulated heterozygous state, obtained by transfecting equal amounts of wild type and mutant cDNAs, showed intermediate behaviour (fig 3A). In addition, the mutant isoform showed rapid mortality typical of cells lacking Na,K ATPase pump activity (fig 3B).

To verify the expression constructs, we tested the wild type and mutant constructs by in vitro transcription and translation experiments. All the constructs gave the expected $112 \mathrm{kDa}$ protein band, therefore excluding that a cloning artefact is responsible for the vulnerability of HeLa cells to ouabain when transfected with the mutant ATPIA2 cDNAs.

To confirm that the mutant AHC isoform is correctly located in the plasma membrane, COS-7 cells were transfected with the wild type and the mutated constructs carrying an $\mathrm{N}$-terminus myc tag. Immunofluorescence experiments show the same pattern of plasma membrane and cytoplasmic localisation for the wild type and the mutant isoform, thus excluding a possible retention of the mutant in the endoplasmic reticulum or other compartments. In addition, Western blot performed on microsomal and cytosolic fractions indicated that both the wild type and the AHC mutant constructs are detectable in the membrane fraction (not shown).

\section{DISCUSSION}

The clinical features of AHC and criteria for diagnosis include: onset before 18 months of age; repeated bouts of 
A
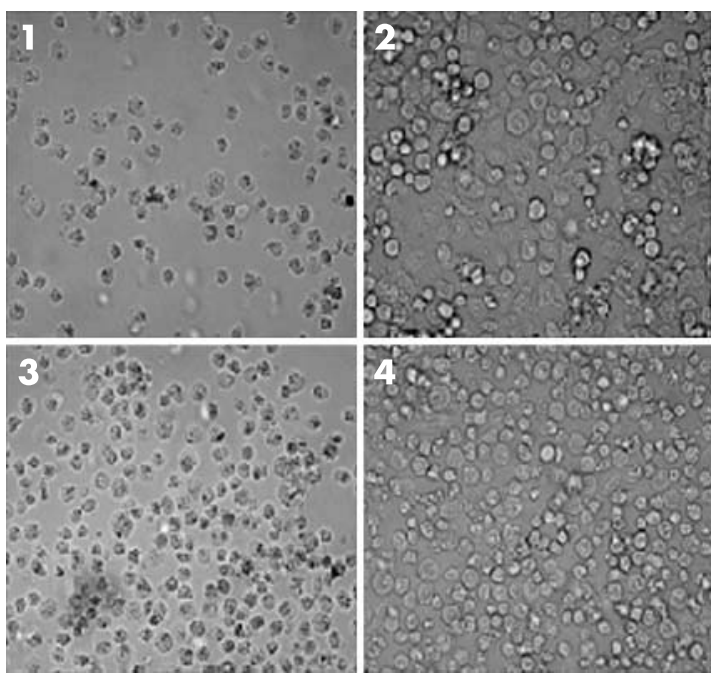

B

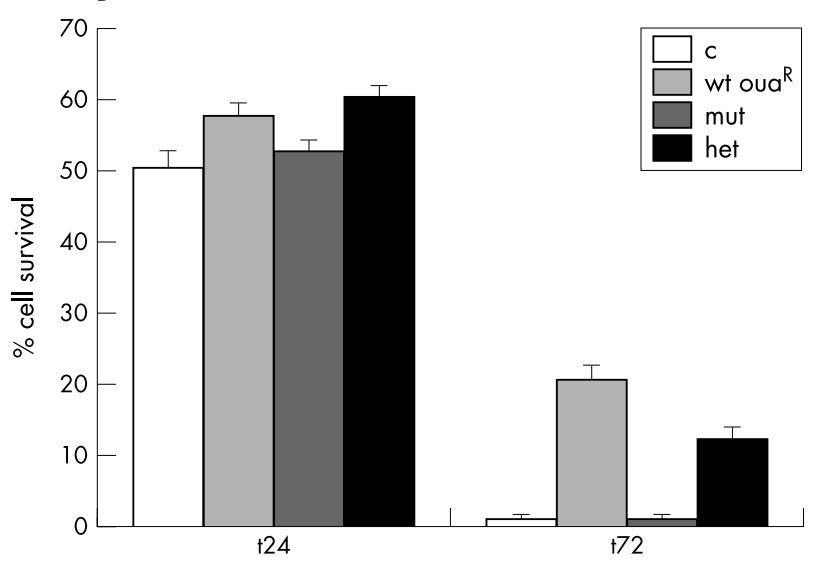

Figure 3 Oubain sensitivity of cells transfected with wild type and mutant ATP1A2 cDNAs. (A) Phase-contrast pictures (magnification $60 \times$ of HeLa cells after $72 \mathrm{~h}$ of treatment with $1 \mu \mathrm{M}$ ouabain and transfected by: 1, a construct expressing non-ouabain-resistant wild type ATP1A2; 2, a construct expressing ouabain-resistant wild type ATP1A2; 3, a construct expressing ouabain-resistant T378N ATP1A2 mutant; 4, a 1:1 mix of constructs expressing ouabain-resistant wild type and T378N ATP1A2 mutant to simulate the heterozygous state. (B) Graphic representation of cell viability after 24 and $72 \mathrm{~h}$ of ouabain treatment.

hemiplegia of varying intensity involving either or both sides of the body at least in some attacks; hemiplegic attacks precipitated by various factors including emotional triggers, head trauma, and fatigue; paroxysmal phenomena including tonic spells, dystonic posturing, choreoathetoid movements, nystagmus or other ocular abnormalities, and autonomic disturbances during and between hemiplegic attacks; attacks terminated by sleep; evidence of progression of mental and neurological deficits (reviewed in Rho and Chugani ${ }^{2}$ and Aicardi et $a^{3}{ }^{3}$ ). Apart from one case reported by Saito et al, ${ }^{15}$ in which AHC is associated with cerebellar atrophy, CT and MRI scans of the brain in AHC patients usually reveal no abnormalities, so that the sites of origin for the transient and persistent clinical signs remain uncertain. The variability of clinical presentation within familial and sporadic cases has always made diagnosis of the disorder particularly difficult. Therefore, for sometime, neurologists considered AHC a migraine equivalent, ${ }^{1}$ an unusual form of epilepsy, or a movement disorder. Problems of misdiagnosis and under-diagnosis could also partially account for the apparent low prevalence of this disorder.

In the first familial case reported, ${ }^{8}$ with four affected members in two generations, the disease was characterised by unilateral and bilateral hemiplegic attacks, dystonia, ocular motor abnormalities, and disappearance of the symptoms with sleep. However, in contrast to the situation in most sporadic cases, onset was later than usual in two members and the oldest patient was able to live a relatively independent life as an adult. Overall, the clinical presentation mimicked intractable epilepsy. ${ }^{8}$

The family we are reporting here displays an analogous phenotype variability with all three types of hemiplegic attacks being present within the members of this family, that is, alternating unilateral (mostly in III- 1 and III-3), shifting (II-4), and bilateral (III-4). Age of onset of the first clinical manifestations and of hemiplegic attacks in all patients was within or very close to the defined age range. According to the criteria for AHC diagnosis, dysarthria, autonomic disturbances, and paroxysmal manifestations, such as choreoathetosic movements, during and between the attacks, are present in all affected subjects, with quite a variable degree of severity, being very mild mostly in the mother (individual II-4) and in the fourth son (individual III-4). In particular, the last neurological examination of the youngest subject only evidenced mild mental retardation with learning disabilities and mild dysarthria but no other neurological signs such as nystagmus, which is typical of sporadic cases.

The effect of sleep on termination of the attacks is also compatible with the disease diagnosis. Analogously, normal neuroradiological examination and muscle biopsy (whenever available) support a diagnosis of AHC.

Epileptic seizures are not common in this family, since only one individual (III-3) has shown sporadic generalised tonicclonic seizures apparently unrelated to the hemiplegic bouts.

The lack of neuroradiological data for individuals II-4 and III-4 has hampered a clear and straightforward diagnosis of AHC. However, the shifting attacks in II-4 and the series of apparently mild bilateral attacks in III-4 associated with paroxysmal phenomena, cognitive impairment, and dysarthria favour the hypothesis of alternating hemiplegia. Indeed among the several conditions that mimic AHC, namely Moya Moya disease (MIM 252350), CADASIL (MIM 125310), and FHM (MIM 141500 and 602481), the last one shows the most overlapping phenotype, being characterised by repeated episodes of hemiparesis or hemiplegia, which usually last 30-60 min and are followed by a severe pulsatile headache which lasts a few hours to days. The onset of the attacks is between 5 and 30 years of age, while in AHC it is before 2 years of age. No choreiform movements or dystonic posturing are reported in any of the FHM cases. Moreover, in FHM, migraine usually accompanies hemiplegic attacks, and developmental delay and cognitive impairment are unusual and, when present, mild.

Therefore, overall, all clinical data about our family members support the diagnosis of alternating hemiplegia. However, some clinical features of the present AHC family, such as the very mild phenotype of individuals II-4 and III-4, the few neurological signs in individual III-4, and the frequency of hemiplegic attacks, being in the lowest range (lower/equal than one/month), all together differentiate this family from most sporadic cases previously reported. ${ }^{34}$

In addition, we can detect a direct association between the severity of hemiplegic attacks and the grade of developmental delay and cognitive decline, which represent two of the major manifestations of AHC syndrome. Indeed, although a progressive cognitive decline, associated with the disease, is 
shared by all affected members of the family, it is clearly more severe in individuals III-1 and III-3 who had severe hemiplegic attacks, than in individuals II-4 and III-4.

In conclusion, the clinical findings observed in our AHC family confirm what has been previously described in the first family reported, ${ }^{8}$ a clearly milder phenotype shared by familial cases compared to sporadic cases. However, despite the clinical variability, all affected members of our family share the same novel mutation in the ATPIA2 gene.

The Na,K ATPase is a heteromeric enzyme comprised of a catalytic $112 \mathrm{kDa} \alpha$ subunit, and $\beta$ and $\gamma$ subunits. Four $\alpha$ subunit isoforms have been identified, each with unique tissue distribution: $\alpha \mathrm{l}$ is ubiquitous, $\alpha 4$ is restricted to testis, $\alpha 3$ is well represented in brain, and $\alpha 2$ is abundant in heart and brain, in both neurons and astrocytes. The AHC mutation described here is located in the largest intracellular loop of the $\alpha 2$ isoform between M4 and M5; this loop represents an important regulatory region for the ion transport system, harbouring the ATP-binding and hydrolasic domains. More precisely, the AHC mutation involves a highly conserved sequence, DKTGTL, which is the target of the $\beta$-aspartyl-phosphorylation reaction during catalysis in all P-type E1-E2-ATPases independently of their cation specificities. ${ }^{16} 17$

According to this, the AHC mutation appears to cause a loss of function effect when transfected in HeLa cells, despite its correct localisation in the plasma membrane. Interestingly, a mutation in the same highly conserved esapeptide sequence was found in the sarco-endoplasmic reticulum $\mathrm{Ca}^{2+}$ ATPase type 2 gene $(A T P 2 A 2)$ leading to Darier disease. ${ }^{18}$ The authors suggest that mutations in the ATP2A2 gene produce a dominant phenotype through haploinsufficiency.

Mutations in the ATPIA2 gene, but not in the same phosphorylation consensus sequence, have previously been recognised as responsible for familial hemiplegic migraine type II (FHM2), a disease with symptoms partially overlapping those of $\mathrm{AHC}^{11}$ and $\mathrm{FHM} 2$ associated with benign familial infantile convulsions (BFIC). ${ }^{12}$ Also the FHM2 mutations described so far seem to have a loss of function effect when tested in eucaryotic cells, indicating that haploinsufficiency of ATPIA2 caused by loss of a single allele leads to FHM2. The pathogenic mechanism leading to aura and migraine in FHM2 might involve two synergistic events: an increase in extracellular $\mathrm{K}^{+}$, producing wide cortical depolarisation, and an increase in intracellular $\mathrm{Na}^{+}$, which enhances intracellular $\mathrm{Ca}^{2+}$ through a decreased $\mathrm{Na}-\mathrm{Ca}$ exchanger function. Supporting this is evidence that the $\alpha 2$ subunit distribution on the plasma membrane coincides with the localisation of the $\mathrm{Na}-\mathrm{Ca}$ exchanger in neurons and astrocytes. ${ }^{19}$ At this point, the hypothesis formulated by Lance $^{20}$ of AHC being a variant of migraine, could be better reformulated based on our findings, which demonstrate allelism between AHC and FHM2.

\section{ACKNOWLEDGEMENTS}

We wish to thank the AHC patients, their families, the Italian Association of Alternating Hemiplegia (AISEA), and the Agarini Foundation for their invaluable support of this work. We are also grateful to T Granata, E Veneselli for referral of subjects, ME Raggi for karyotype analysis, R Giorda, MG D'Angelo, L Atorino and L Silvestri for significant contributions and advice.

\section{ELECTRONIC-DATABASE INFORMATION}

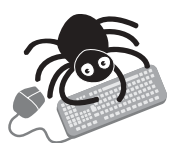

Electronic database information is as follows: Human Genome Browser: http://genome.cse.ucsc.edu/; ATPIA2 cDNA: http://www.ncbi.nlm.nih.gov/

\section{Authors' affiliations}

M T Bassi, N Bresolin, A Tonelli, F Crippa, C Baschirotto, C Zucca, Scientific Institute E. Medea, Bosisio Parini, Lecco, Italy

N Bresolin, K Nazos, A Bersano, IRCCS Ospedale Maggiore Policlinico, Department of Neurological Sciences, University of Milan, Milan, Italy D Dolcetta, F M Boneschi, V Barone, G Casari, Dibit-San Raffaele Scientific Institute, Milan, Italy

GC and VB are supported by the EU "EUROHEAD" grant.

Conflict of interest: none declared.

Correspondence to: $\operatorname{Dr}$ G Casari, Dibit-San Raffaele Scientific Institute, Via Olgettina 58, 20132 Milan, Italy; casari.giorgio@hsr.it

Correspondence to: $\operatorname{Dr} M$ T Bassi, Laboratory of Molecular Biology, Scientific Institute E. Medea, Via D.L. Monza 20, 23842, Bosisio Parini, Lecco, Italy; mtbassi@bp.Inf.it

Received 12 February 2004

Accepted for publication 9 March 2004

\section{REFERENCES}

1 Verret S, Steele JC. Alternating hemiplegia in childhood: a report of eight patients with complicated migraine beginning in infancy. Pediatrics 1971;47:675-80.

2 Rho JM, Chugani HT. Alternating hemiplegia of childhood: insights into its pathophysiology. J Child Neurol 1998;13(1):39-45.

3 Aicardi J, Bourgeois M, Goutieres F. Alternating hemiplegia of childhood: clinical findings and diagnostic. In: Anderman F, Aicardi J, Vigevano F, eds. Alternating hemiplegia of childhood. New York: Raven Press, 1995:3-18.

4 Mikati MA, Kramer U, Zupanc ML, Shanahan RJ. Alternating hemiplegia of childhood: clinical manifestations and long term outcome. Pediatr Neurol 2000;23(1):134-41

5 Silver K, Andermann F. Alternating hemiplegia of childhood: the natural history of the disorder in a group of 10 patients. In: Anderman F, Aicardi J, Vigevano F, eds. Alternating hemiplegia of childhood. New York: Raven Press, 1995: 19-28.

6 Olesen J. Classification and diagnostic criteria for headache disorders. Cephalalgia 1988;8(suppl 7):26.

7 Bourgeois M, Aicardi J, Goutieres F. Alternating hemiplegia of childhood. J Pediatr 1993;122(5 Pt 1):673-9.

8 Mikati MA, Maguire H, Barlow CF, Ozelius L, Breakefield XO, Klauck SM, Korf B, O'Tuama SL, Dangond F. A syndrome of autosomal dominant alternating hemiplegia: clinical presentation mimicking intractable epilepsy; chromosomal studies; and physiologic investigations. Neurology 1992;42(12):2251-7.

9 Kramer U, Nevo Y, Margalit D, Shorer Z, Harel S. Alternating hemiplegia of childhood in half sisters. J Child Neurol 2000;15(2): 128-30.

10 Arnold DL, Silver K, Andermann F. Evidence for mitochondrial dysfunction in patients with alternating hemiplegia of childhood. Ann Neurol 1993;33(6):604-7.

11 De Fusco M, Marconi R, Silvestri L, Atorino L, Rampoldi L, Morgante L, Ballabio A, Aridon P, Casari G. Haploinsufficiency of ATP1A2 encoding the $\mathrm{Na}+/ \mathrm{K}+$ pump alpha2 subunit associated with familial hemiplegic migraine type 2. Nat Genet 2003;33(2):192-6.

12 Vanmolkot KR, Kors EE, Hottenga JJ, Terwindt GM, Haan J, Hoefnagels WA, Black DF, Sandkuijl LA, Frants RR, Ferrari MD, van den Maagdenberg AM. Novel mutations in the Na+, K+-ATPase pump gene ATP1A2 associated with familial hemiplegic migraine and benign familial infantile convulsions. Ann Neurol 2003;54(3) 360-6.

13 Marconi M, De Fusco M, Aridon P, Plewnia K, Rossi M, Carapelli S, Ballabio A, Morgante L, Musolino R, Epifanio A, Micieli G, De Michele G, Casari G. Familial hemiplegic migraine type 2 is linked to $0.9 \mathrm{Mb}$ region on chromosome lq23. Ann Neurol 2003;53. 376-81.

14 Hu YK, Kaplan JH. Site-directed chemical labeling of extracellular loops in a membrane protein. The topology of the Na,K-ATPase $\alpha$ subunit. J Biol Chem 2000;275: 19185-91.

15 Saito Y, Sakuragawa N, Sasaki M, Sugai K, Hashimoto T. A case of alternating hemiplegia of childhood with cerebellar atrophy. Pediatr Neurol 1998;19(1):65-8.

16 Kuntzweiler TA, Wallick ET, Johnson CL, Lingrel JB. Amino acid replacement of Asp369 in the sheep alpha 1 isoform eliminates ATP and phosphate stimulation of $\left[{ }^{3} \mathrm{H}\right]$ ouabain binding to the $\mathrm{Na}+, \mathrm{K}(+)$-ATPase without altering the cation binding properties of the enzyme. J Biol Chem 1995;270(27): 16206-12. 
17 Pedersen PA, Rasmussen JH, Jorgensen PL. Consequences of mutations to the phosphorylation site of the alpha-subunit of $\mathrm{Na}, \mathrm{K}$-ATPase for ATP binding and E1-E2 conformational equilibrium. Biochemistry 1996:35(50): 16085-93.

18 Sakuntabhai A, Ruiz-Perez V, Carter S, Jacobsen N, Burge S, Monk S Smith M, Munro CS, O'Donovan M, Craddock N, Kucherlapati R, Rees JL, Owen M, Lathrop GM, Monaco AP, Strachan T, Hovnanian A. Mutations in
ATP2A2, encoding a $\mathrm{Ca}^{2+}$ pump, cause Darier disease. Nat Genet 1999;21(3):271-7.

19 Juhaszova $\mathrm{M}$, Blaustein MP. $\mathrm{Na}^{+}$pump low and high ouabain affinity alpha subunit isoforms are differently distributed in cells. Proc Natl Acad Sci U S A 1997;94(5):1800-5.

20 Lance JW. Is alternating hemiplegia of childhood (AHC) a variant of migraine? Cephalalgia 2000;20(8):685.

\section{Gene effects in environmentally determined subgroups}

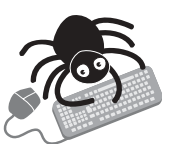

Please visit the Journal of Medical Genetics website [www. jmedgenet. com] for a link to the full text of this article.
$T$ here has been controversy about the usefulness of stratification by environmentally determined subgroups in genetic research. In cancer epidemiology only a few genetic polymorphisms cause a substantial change in risk and many genes involved in the metabolism of mutagens have little or no effect. But the inclusion of environmental measures in genetic studies might reveal environment-gene interactions. Such combined studies have been questioned, however, on the grounds that they are unlikely to increase the power of the genetic study very much. It has been said that the effects of genes in different environmental subgroups are likely to be in the same direction even if varying in size, and this would limit the gain in power. Australian authors have listed several examples to counter this argument.

The first example they give is of the influence of maternal smoking on the effect of the CYPIAl gene on birthweight. The gene controls the metabolism of chemicals in tobacco smoke. When all 741 mothers in a study were considered the incidence of low birthweight was identical $(21.5 \%)$ in mothers with genotype AA and mothers with genotype Aa or aa. Among the 124 maternal smokers however, the incidence of low birthweight was 24\% (AA) $v 45 \%$ (Aa or aa).

The second example concerns the GSTPI glutathione transferase gene, Parkinson's disease, and exposure to pesticides. Among all subjects in one study there was no significant association between the genotype and Parkinson's disease but among subjects exposed to pesticides one polymorphism increased the risk of Parkinson's disease more than fivefold. In the third example they quote a study of the effect of a monoamine oxidase A polymorphism on antisocial behaviour. It has been found that the polymorphism increases the rate of antisocial behaviour only among people who suffered from maltreatment as children. Among people who had not been maltreated in childhood the polymorphism tended to be associated with less antisocial behaviour. Another example of a change in direction of gene effect according to environmental subgroup is the effect of an alcohol dehydrogenase polymorphism on HDL cholesterol concentrations according to level of alcohol consumption. Different polymorphisms may also have different effects on the outcome of a single environmental factor. An example of this is the effect of different $\beta_{2}$ adrenoceptor gene polymorphisms on the risk of obesity at varying levels of carbohydrate consumption.

The authors of this paper conclude that inclusion of environmental data may enhance the search for disease causing genes.

A Journal of Epidemiology and Community Health 2004;00:000-000 